\title{
X-ray perspective on young stellar populations in objects with nuclear activity - the case of our Galactic Center-
}

\author{
Katsuji Koyama, Atsushi Senda and Shin-Ichiro Takagi \\ Department of Physics, Kyoto University \\ email: koyama@cr.scphys.kyoto-u.ac.jp
}

\begin{abstract}
Although our Galactic Center harbors a black hole $\left(\operatorname{Sgr} \mathrm{A}^{*}\right)$ of a few million solar masses, it and its environments are very quiet at present. In X-rays however, the close vicinity of Sgr $\mathrm{A}^{*}$ shows very unique and various phenomena mostly originated from young stellar populations. We report on the X-ray perspective on the young stellar populations which are related to our Galactic Center activities. The discussion is essentially based on the observational facts of new X-ray objects in the Galactic Center region in the $1^{\circ} \times 2^{\circ}$ area. They are;

1) Clusters of young high mass stars, which are Sgr B2, Arches, IRS 13 and Quintuplet.

2) X-ray reflections in the giant molecular clouds, such as Sgr B2, Sgr C, M0.01-0.09 and others.

3) New candidates of X-ray supernova remnants (SNRs), which are Sgr A East, G0.570-0.018 and G359.8-0.3.

4) Non-thermal Jets, Filaments and Shells, which are unique X-ray features in the GC region.

These X-ray features may be closely related with each other, hence may have common origins. A unified picture is presented for the X-ray activity of our Galactic Center comparing with the $\mathrm{X}$-ray spectra from other type of galaxies such as;
\end{abstract}

5) Star burst galaxy (NGC 253), low luminosity AGN (M 81) and Seyfert 2 (NGC 1068).

Keywords. X-rays, The Galactic Center, High mass young stars, Young SNRs, Non-thermal jets and filaments, X-ray reflection nebula.

\section{Introduction}

High resolution infrared astrometry over a decade on the Galactic Center (GC) region detected orbital motions of a number of stars around Sgr A* (Schoedel et al. 2002). The orbital motions directly constrain the mass of $\mathrm{Sgr} \mathrm{A}^{*}$ to be about 3 millions of sun, hence support that the GC harbors a massive black hole. However no clear Xray activity, commonly associated to a massive black hole, has been reported so far. A breakthrough is the discovery of highly ionized iron lines extending around the GC. Likely origin of this emission is a high temperature $\left(10^{7}-10^{8} \mathrm{keV}\right)$ plasma due possibly to violent explosions in the GC region within the past $10^{5}$ years (Koyama et al. 1989). The first hard X-ray imaging satellite, $A S C A$ obtained a high quality spectrum of this plasma resolving the iron lines to $\mathrm{H}$-like and He-like irons. The intensity ratio of these two highly ionized iron lines gives clear evidence for high temperature plasma of 5-10 $\mathrm{keV}$. In addition, $A S C A$ found strong neutral iron lines at $6.4 \mathrm{keV}$ associated with giant molecular clouds (Koyama et al. 1996). The spectrum and morphology are explained to be fluorescence irradiated by strong X-rays (hence: X-ray reflection nebula) coming from the Sgr $\mathrm{A}^{*}$ side. Recent Chandra observations on the GC have confirmed these early results and furthermore, with its unprecedented spatial resolution, have resolved 

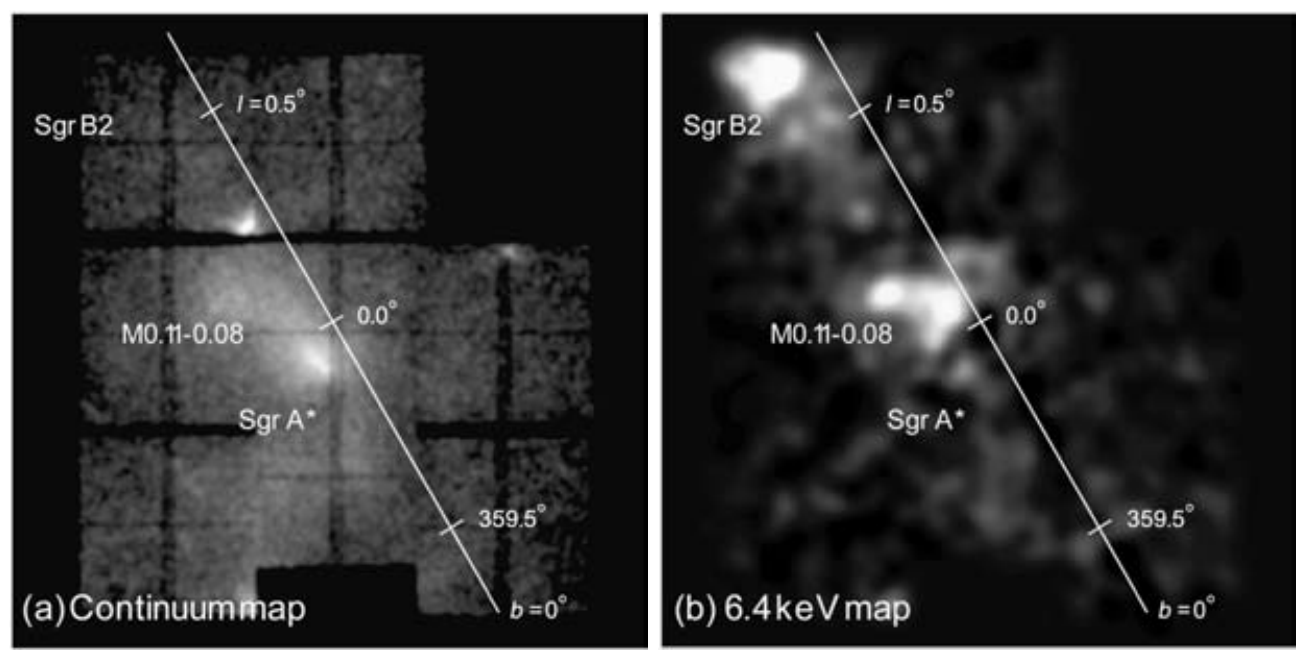

Figure 1. Left: The X-ray image of the Galactic Center region in 2-10 keV. Right: Same as the left, but in $6.4 \mathrm{keV}$ (adopted from Koyama et al. 1996)
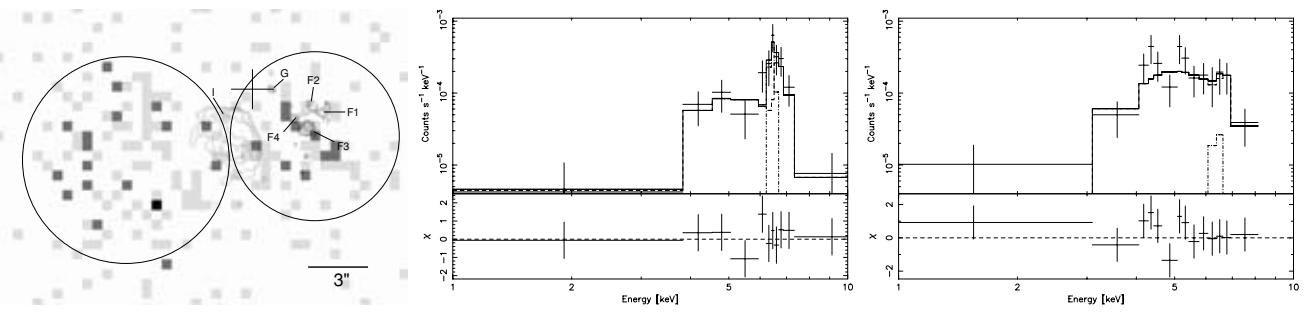

Figure 2. Left: The Chandra X-ray image of the ultra compact HII complex, Sgr B2 Main (contour). The X-ray bin size is $0.5^{\prime \prime} \times 0.5^{\prime \prime}$. Middle: The X-ray spectrum and the best-fit model (solid histogram) of a thin thermal plasma with the $6.4 \mathrm{keV}$ line (the dotted histogram) for No 10. Right: Same as No 10 but for No 13. (adopted from Takagi, Murakami \& Koyama 2002)

a number of SNRs, clusters of high mass stars, non-thermal X-ray filaments as well as many other 6.4-keV clouds (Murakami et al. 2000, Baganoff et al. 2001, Murakami et al. 2001a, Murakami, Maeda \& Koyama 2001b, Murakami 2001c, Wang et al. 2002a, Wang et al. 2002b, Baganoff et al. 2003, Sakano et al. 2003, Park et al. 2004, Muno et al. 2004, Senda 2004). This paper reviews all these observational facts and tries to draw a unified picture of our GC activities.

\section{High Mass Star Forming Regions}

\subsection{The Sgr B2 cloud}

The Sgr B2 cloud, one of the largest giant molecular clouds in our Galaxy, is located at a projected distance of $100 \mathrm{pc}$ from $\mathrm{Sgr} \mathrm{A}^{*}$. The radio continuum band observations have revealed nearly 60 ultra compact (UC) HII regions lying north-to-south (Gaume \& Claussen 1990). Bipolar outflows are found from two of the HII complexes, Sgr B2 Main and North (Lis et al. 1993). Clusters of $\mathrm{OH}, \mathrm{H}_{2} \mathrm{O}$ and $\mathrm{H}_{2} \mathrm{CO}$ masers are found near these HII regions (Mehringer, Goss, \& Palmer 1994). Therefore the cores of Sgr B2 may harbor clusters of high mass stars in a zero-age main-sequence (ZAMS) phase.

Chandra observed Sgr B2 and found more than a dozen X-ray sources, some are at the positions of UCHII complexes (Takagi, Murakami \& Koyama 2002). Figure 2 (Left) 
shows the Chandra X-ray image in the $2-10 \mathrm{keV}$ band overlaid on the radio contours of the ultra compact (UC) HII complex, Sgr B2 Main. The UC HII complex comprises several components, F1-F4, G (De Pree, Goss \& Gaume 1998) and I (Gaume \& Claussen 1990). The cross $(+)$ indicates the position and $1 \sigma$ errors $\left(\sim 1^{\prime \prime}\right)$ of a mid-infrared source. The X-ray complex No 10 is extended along the UC HII "island" F3, F4 and G, hence would be comprised of several point sources, possibly high mass stars. In detail, not all the individual X-ray sources coincide to the UC HIIs. Therefore, these X-ray members would be younger than the phase to ionize the circumstellar medium, or ZAMS. Hence the ages of these stars would be well below $10^{5}$ year.

No 13 is also a complex of X-ray sources lying in the east of the UC HII region I, but is associated with no UC HII region. This may indicate that all the high mass members in No 13 are in a pre-phase of UC HII formation, possibly younger than No 10. Still it exhibits X-rays with comparable luminosity to No 10.

Figure 2 (Middle and Right) shows the X-ray spectra, and the best-fit thin thermal models. The best-fit absorptions are $4 \times 10^{23} \mathrm{Hcm}^{-2}$, one of the largest among the known $\mathrm{X}$-ray emitting stars, indicating that they are really lying at/near the center of the cores of Sgr B2. This demonstrates that hard X-rays are very powerful to discover deeply embedded stars even if they suffer from a large optical extinction of $A \mathrm{v} \sim 200-300 \mathrm{mag}$. The absorption corrected luminosities in the $2-10 \mathrm{keV}$ band for No 10 and No 13 are $8 \times 10^{32} \mathrm{ergs} \mathrm{s}^{-1}$ and $13 \times 10^{32} \mathrm{ergs} \mathrm{s}^{-1}$, respectively. As already noted, these X-ray sources are likely to be complexes of several high mass stars, hence the luminosity of the individual high mass star would be in the order of $10^{32} \mathrm{ergs} \mathrm{s}^{-1}$. This is similar value to the nearest high mass young stellar object(YSO), $\theta$ C Ori of spectral type O6-7 (Schulz et al. 2001) and larger than those of high mass young stars in the Monoceros R2 cloud (Kohno, Koyama \& Hamaguchi 2002).

A notable feature of No 10 is strong lines at $6.7 \mathrm{keV}$ and $6.4 \mathrm{keV}$. The former $(6.7 \mathrm{keV}$ line) is the K-shell transition line from He-like irons in thin hot plasma. The latter (6.4 keV line) may be due to neutral or low-ionization irons in a dense circum/inter stellar medium irradiated by the central stars. The iron abundance determined from both of the lines is more than 5 times solar, which is significantly larger than that from any other high mass star forming region (Kohno, Koyama \& Hamaguchi 2002, Schulz et al. 2001, Yamauchi et al. 1996). The iron abundance of No 13, in contrast to No 10, is sub-solar.

\subsection{The Arches and Quintuplet Clusters}

The Arches Cluster is also a giant molecular cloud located near Sgr A* with young stars of ages about 2 Myr. The Chandra observations were made in 2001 and 2004, and found three bright X-ray sources at the position of the IR high mass stars No 21, 23 and 26 (Blum et al. 2001). No 23 and No 21 are also radio sources AR1 and AR4, hence would have large mass loss of $1.7 \times 10^{-4}$ and $3.9 \times 10^{-5} \mathrm{M}_{\odot}$, respectively (Lang, Goss \& Rodríguez 2001). Figure 3 (Left) is the Chandra X-ray image made by the observation in 2004. The X-ray spectra of the three stars are fitted with a thin thermal model. They all show large X-ray absorptions of $\sim 10^{23} \mathrm{H} \mathrm{cm}^{-2}$, which is consistent with being the cluster members. The X-ray luminosities in the $2-10 \mathrm{keV}$ band are $2-5 \times 10^{33} \mathrm{ergs} \mathrm{s}^{-1}$, one of the highest among any known young stars.

A unique feature is a diffuse emission elongated to the southeast of the cluster core. The X-ray spectrum shows a strong iron line at $6.4 \mathrm{keV}$. The morphology and spectral features indicate that the diffuse emission is due to particle or X-ray irradiation from the direction of the cluster core. If the source is X-ray, the required luminosity must be a few $\times 10^{35} \mathrm{ergs} \mathrm{s}^{-1}, 10$ times brighter than the present value. One possibility is that the 

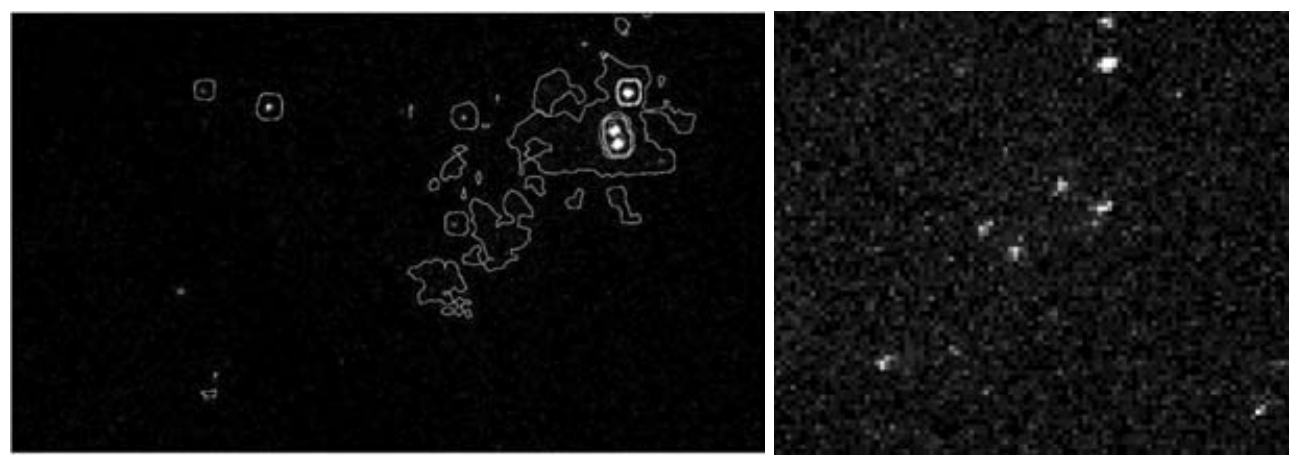

Figure 3. The 2-10 keV band X-ray image of the Arches Cluster (Left) and the Quintuplet Cluster (Right).

Arches cluster was brighter than the present value in the near past. It should be noted, however, no large flux variation is found between the two observations in 2001 and 2004. Another possibility is a jet of high energy particles emanating from the core, because the diffuse emission is collimated to one direction. We note that some young stars emit fairly high speed jets in a very early stage of the star evolution (e.g. Tsujimoto et al. 2004). The Arches young stars may have fur active jets, which may be related to extreme physical conditions (e.g., high magnetic field, high density, etc.) in the GC region.

The Quintuplet cluster is located nearly the same distance as the Arches cluster, but is more evolved with estimated age of about $4 \mathrm{Myr}$. This cluster also includes many bright IR sources, possibly high mass young stars. The Chandra X-ray image in Figure 3 (Right) shows several point sources, but only 2 sources are clearly associated to the IR stars (No. 231 and No. 211 in Figer, McLean \& Morris 1999). The luminosity of the point sources is in the order of $10^{32} \mathrm{erg} \mathrm{s}^{-1}$, which is not unusually high, but is typical to normal young high mass stars.

\subsection{The GC cluster: IRS $13 E$}

Many IR star clusters have been reported in the close vicinity of Sgr A*. Although Chandra found many moderately bright X-ray sources, possible X-ray association to the IR clusters is only IRS 13E (Baganoff et al. 2003). The X-ray luminosity is in the order of $10^{33} \mathrm{erg} \mathrm{s}^{-1}$, which is typical to clusters of young high mass stars. Morris et al. (2002) pointed out that a jet-like structure is extending to the north, similar morphology to that of the Arches cluster. Unlike Arches however, the "jet" spectrum is featureless, nonthermal typical to relativistic jets from black holes, for example. Schoedel et al. (2005) argued that IRS 13E must include an intermediate mass black hole to be bounded against the large tidal force of Sgr A*. With the available data however, it is hard to conclude whether the jet-like structure is real jet emanating from IRS $13 \mathrm{E}$, or only a series of X-ray sources accidentally aligned.

\section{The X-ray Reflection Nebula}

$A S C A$ found that the narrow band $\mathrm{X}$-ray image at $6.4 \mathrm{keV}$ in the $\mathrm{GC}$ region is totally different from that of the wide band image (see Figure 1). Figure 1 (Right) shows the $A S C A 6.4 \mathrm{keV}$ band image. Clumpy structures on the giant molecular cloud Sgr B2, M0.01-0.09 and others are found. These $6.4 \mathrm{keV}$ clumps are confirmed by Chandra with high resolution imaging. Since Sgr B2 is most deeply observed, we concentrate on Sgr B2 and discuss the origin. 

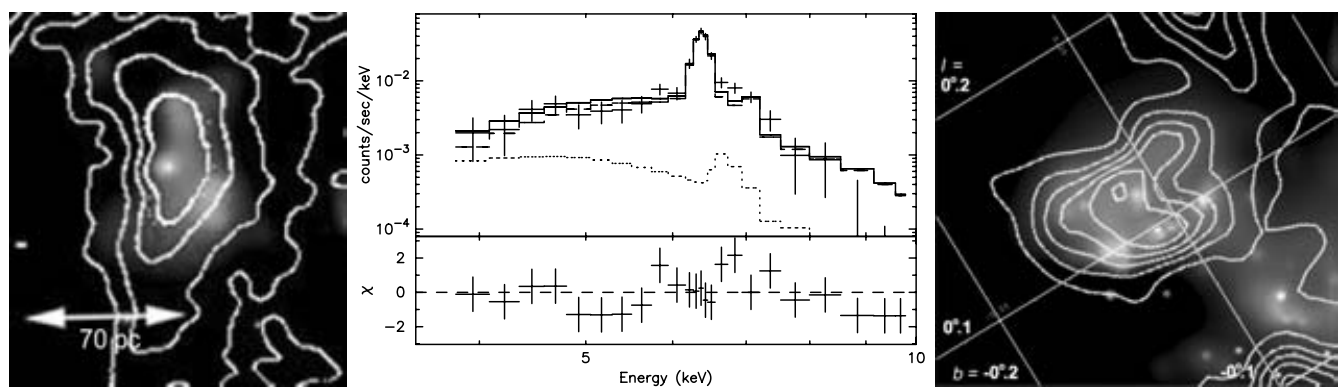

Figure 4. Examples of the X-ray reflection nebulae; Left: The $6.4 \mathrm{keV}$ image of Sgr B2 overlaid on the radio contour. Middle: The spectrum of Sgr B2. Right: The $6.4 \mathrm{keV}$ image of M0.01-0.09 overlaid on the radio contour (adopted from Murakami, Maeda \& Koyama 2001b and Murakami 2001c).

Figure 4 (Left) is the Chandra image in $6.4 \mathrm{keV}$ overlaid on the radio contours of Sgr $\mathrm{B} 2$. We see a crescent shape on the cloud in the near side of the GC and the convexed side pointing to the GC direction. The X-ray spectrum (Figure 4, Middle) shows a strong $6.4 \mathrm{keV}$ line and a sharp edge at $7.1 \mathrm{keV}$. These structures can only be made by the irradiation of external X-rays on the neutral gas. The crescent morphology with the convexed side pointing to the GC direction also supports that the external X-rays are coming from the GC direction.

To demonstrate this scenario, we made a simulation. The simulated X-ray spectrum is shown by the solid line of Figure 4 (Middle). The morphology and spectrum can be well reproduced by the simulation, supporting the X-ray reflection scenario with external Xray sources in the GC direction. Thus we call the $6.4 \mathrm{keV}$ clump as the X-ray Reflection Nebula. Since no bright source is found near the GC region, we infer that the irradiation source is $\mathrm{Sgr} \mathrm{A}^{*}$, which is presently inactive but was active about 300 years ago, the light traveling time from Sgr A* to Sgr B2.

Although not as clear as the case of the Sgr B2 cloud, we found many $6.4 \mathrm{keV}$ clumps in the close vicinity of the GC (Bamba et al. 2002). If not all, these clumps may be also made by the past X-ray activity of Sgr $\mathrm{A}^{*}$. Using the reflection nebula scenario, and using the possible distance from $\operatorname{Sgr} \mathrm{A}^{*}$ to the reflection nebulae, we can infer that the past X-ray flux of Sgr A* has monotonously declined to the present value (Murakami 2001c).

\section{Supernovae and the Remnants}

Since the GC region is filled with the X-ray emitting plasma, X-rays due to possible SNRs are hidden in this high X-ray background. Still we discovered several SNR candidates with a thin thermal spectrum (Senda 2004). With ASCA, Chandra and XMMNewton, we found a ring-like structure (G359.8-0.3) at the south of the GC. This source shows emission lines from highly ionized $\mathrm{Si}$ and $\mathrm{S}$. The temperature is about $0.5 \mathrm{keV}$, hence may be a new SNR. Near the west of Sgr B2, we found a ring-and-tail structure (G0.570-0.018). The spectrum shows a $6.5-6.6 \mathrm{keV}$ line, indicating very hot plasma (a few $\mathrm{keV}$ ). This also may be a candidate young SNR (Senda 2004).

The most important fact is the confirmation of an energetic SNR associated to the radio shell Sgr A East (Maeda et al. 2002). The X-ray spectrum shows highly ionized $\mathrm{Si}, \mathrm{S}, \mathrm{Ar}$ and Fe lines, of which Fe is clearly over-abundant. The intensity ratio of Helike and H-like iron lines give the plasma temperature of about $4 \mathrm{keV}$ (Sakano et al. 2003). Therefore, Sgr A East must be very young SNR with the age of about 1000 year. 

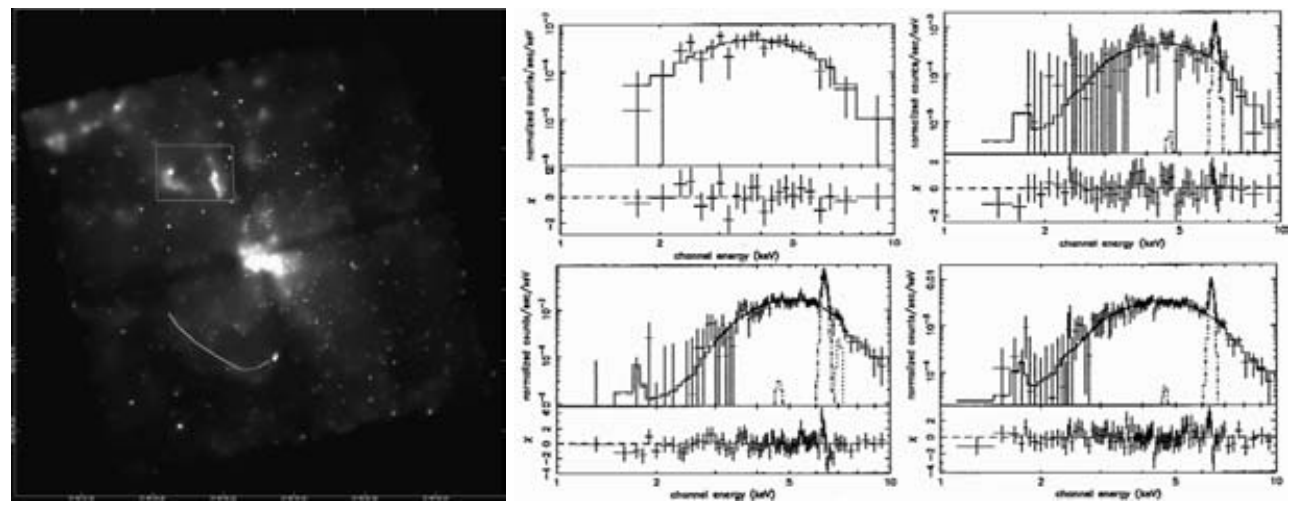

Figure 5. Left: The 3-color X-ray image near the GC region: $r e d=0.5-2 \mathrm{keV}$, blue $=2-6 \mathrm{keV}$. green $=6.4 \mathrm{keV}$. Upper left is a cluster of X-ray reflection nebulae, while lower left is a non-thermal arc (the blue line, see text). Right: Examples of spectra from the X-ray Reflection Nebulae shown in the Left Figure (adopted from Senda 2004).

Interesting feature of this SNR is a bow-structure with a point source at the north head (Park et al. 2004). The spectrum of the point source is a power-law, which resembles to a pulsar. Although no pulsation is found, the point source would be a young neutron star kicked-off by the supernova explosion.

At the southeast from Sgr A*, we see an arc-structure (see Figure 5, Left). This arc may be a part of SNR or a relic of a possible outburst of the GC. Interestingly the spectrum is non-thermal like SN 1006 (Koyama et al. 1995). We will discuss the non-thermal X-rays in more detail in the following section.

\section{Non-Thermal Knots, Jets and Filaments}

In the GC region, there are many knots, or filaments with a non-thermal spectrum (see Figures 6 and 7, and also Morris et al. 2002). In Figure 6, we see three elliptical knots on a line going to the northwest from Sgr A*. The major axis of the ellipses are also aligned on this line. Thus the three knots are likely ejected from the GC in several 100 years ago. Most of the other knots and filaments are inside or near the boundary of the SNR Sgr A East, hence may be related to the SN explosion. However, it is not clear whether they are ejected from Sgr A East or Sgr A*, or due to other origins.

From the reflection nebulae, non-thermal jets and the supernova explosion of Sgr A East, we infer a unified model of our Galactic Center activity. As already pointed out a supernova explosion occurred at the center of Sgr A East about 1000 year ago. This made an expanding shocked shell. About 300 years ago, the shocked shell arrived at Sgr $\mathrm{A}^{*}$, the super massive black hole. Since the shocked shell has higher density than the GC environments, a larger mass accretion to Sgr A* had occurred. This produced bright $\mathrm{X}$-rays and ejection of high speed jets.

\section{What is our Galactic Center ?}

As we have been discussing, many categories of X-ray objects are contributing to the GC activity. If our GC is at a long distance, for example $10-20 \mathrm{Mpc}$, we can not resolve individual objects in the GC, but can see it as a point or a small spot like the center of other galaxies. The GC spectrum, which includes all the relevant objects, is shown in Figure 8 (Left). A question is "who has a similar spectrum ?" We compare the X-ray 


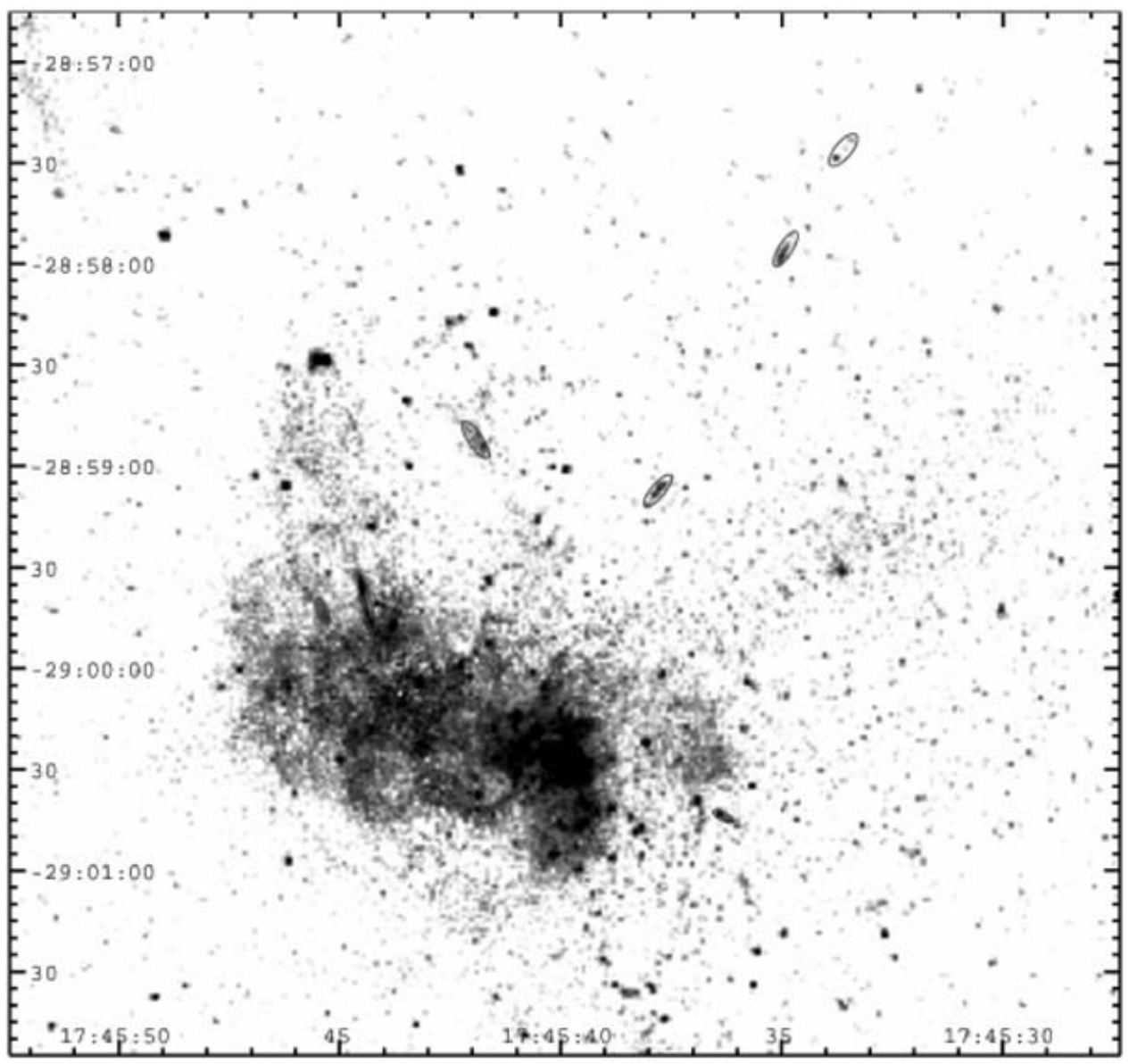

Figure 6. Non-thermal jets, arcs and filaments around Sgr A* (green ellipses). Three knots which are aligned on a line toward the northwest direction would be X-ray jets (adopted from Senda 2004).
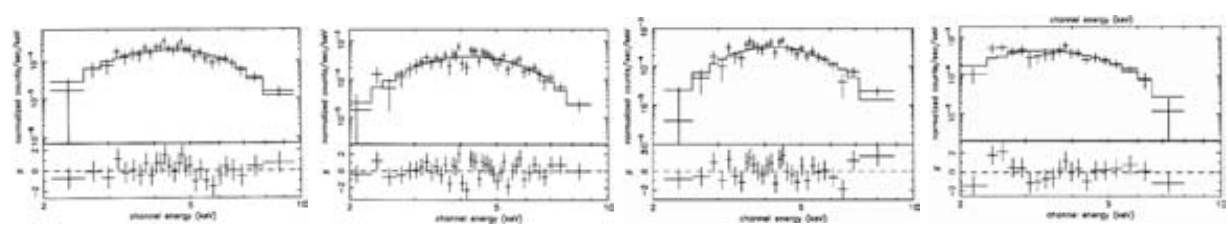

Figure 7. Examples of non-thermal spectra for the four nearest knots from Sgr A* (adopted from Senda 2004).

spectra of the centers of different types of galaxies, M31 (twin of the Milky Way), M81 (low luminosity AGN), NGC 253 (starburst galaxy) and NGC 1068 (Seyfert2 galaxy) (Takahashi et al. 2004, Pietsch et al. 2001, Page et al. 2004, Weaver et al. 2002, Matt et al. 2004). Among them, the NGC 1068 spectrum is very similar to the GC, but its absolute luminosity is far larger. NGC 1068 has a very active nucleus, but is blocked by a dense torus, and what we observe is the reflected component. Also the center of NGC 1068 is currently in a very active star forming phase. Thus the spectrum of NGC 1068 is a combination of the reflected AGN and the starburst activity. Our Galactic Center must be a scale-down version of NGC 1068. The essential difference is that NGC 1068 

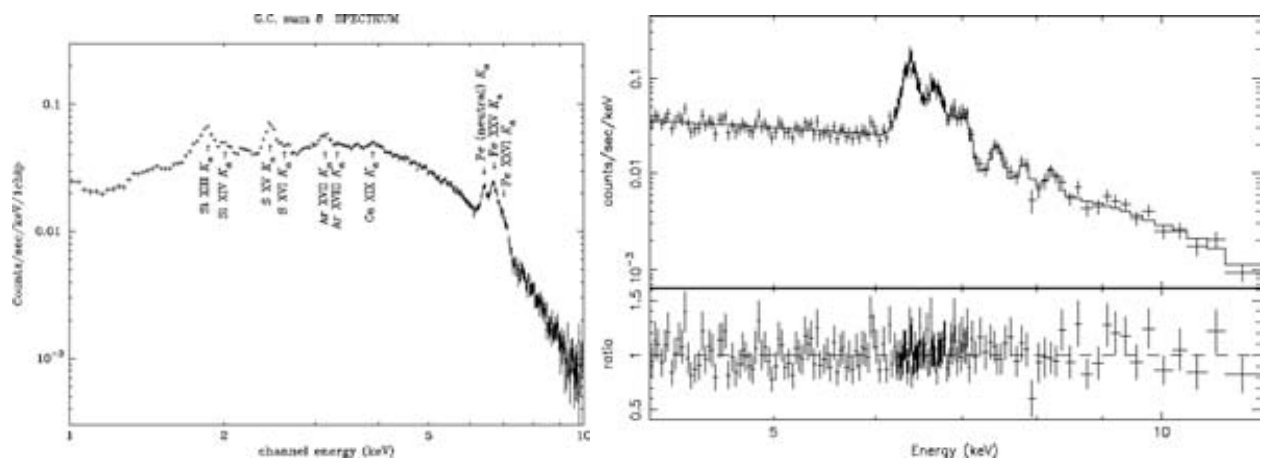

Figure 8. Comparison of the Galactic center spectra of the Milky Way (Left) and the Seyfert 2 galaxy NGC 1068 (Right) (adopted from Koyama et al. 1996 and Matt et al. 2004).

presently has an active nucleus but is hidden. The supper massive black hole in our Galactic Center is not active at present, but was active in the past and the reflected $\mathrm{X}$-rays have just now arrived at the Earth.

\section{Acknowledgements}

We would like to acknowledge Drs Maeda and Murakami, who provide the ASCA and Chandra results. This work is supported by the Grant-in-Aid for the 21st Century COE "Center for Diversity and Universality in Physics" from the Ministry of Education, Culture, Sports, Science and Technology (MEXT) of Japan.

\section{References}

Baganoff, F. K., Bautz, M. W., Brandt, W. N., Chartas, G., et al. 2001, Nature, 413, 45

Baganoff, F. K., Maeda, Y., Morris, M., Bautz, M. W., et al. 2003, ApJ, 591, 891

Bamba, A., Murakami, H., Senda, A., Takagi, S. Yokogawa, J. \& Koyama, K. 2002, Proceedings of the Symposium "New Visions of the X-ray Universe in the XMM-Newton and Chandra era", Noordwijk, NL

Blum, R. D., Schaerer, D., Pasquali, A., Heydari-Malayeri, M., Conti, P. S. \& Schmutz, W. 2001, AJ, 122, 1875

De Pree, C. G., Goss, W. M. \& Gaume, R. A. 1998, ApJ, 500, 847

Figer, D. F., McLean, I. S. \& Morris, M. 1999, ApJ, 514, 202

Gaume, R. A. \& Claussen, M. J. 1990, ApJ, 351, 538

Kohno, M., Koyama, K.\& Hamaguchi, K. 2002, ApJ, 567, 423. also Erratum in ApJ, 580, 626

Koyama, K., Awaki, H., Kunieda, H., Takano, S. \& Tawara, Y. 1989, Nature, 339, 603

Koyama, K., Petre, R., Gotthelf, E. V., Hwang, U., Matsuura, M., Ozaki, M. \& Holt, S. S. 1995, Nature, 378, 255

Koyama, K., Maeda, Y., Sonobe, T., Takeshima, T., Tanaka, Y. \& Yamauchi, S. 1996, Publ. Astron. Soc. Japan, 48, 249

Lang, C. C., Goss, W. M. \& Rodríguez, L. F. 2001, ApJ (Letter), 551, L143

Lis, D. C., Goldsmith, P. F., Carlstrom, J. E. \& Scoville, N. Z. 1993, ApJ (Letter), 402, L238

Maeda, Y., Baganoff, F. K., Feigelson, E. D., Morris, M. et al. 2002, ApJ, 570, 671

Matt, G., Bianchi, S., Guainazzi, M. \& Molendi, S. 2004, A\& A, 414, 155

Mehringer, D. M., Goss, W. M. \& Palmer, P. 1994, ApJ, 434, 237

Morris, M., Baganoff, F., Muno, M., Howard, C. et al. 2002, Proceedings of the Galactic Center Workshop, 167, Hawaii, USA

Muno, M. P., Baganoff, F. K.. Bautz, M. W., Feigelson, E. D. et al. 2004, ApJ, 613, 326

Murakami, H., Koyama, K., Sakano, M. Tsujimoto, M. \& Maeda, Y. 2000, ApJ, 534, 283

Murakami, H., Koyama, K., Tsujimoto, M., Maeda, Y. \& Sakano, M. 2001a, ApJ, 550, 297

Murakami, H., Koyama, K. \& Maeda, Y. 2001b, ApJ, 558, 687 
Murakami, H. 2001c, PhD thesis, Kyoto University

Page, M. J., Soria, R., Zane, S., Wu, K. \& Starling R. L. C. 2004, A\&A, 422, 77

Park, S., Muno, M. P., Baganoff, F. K., Maeda, Y. et al. 2004, ApJ, 603, 548

Pietsch, W., Roberts, T. P., Sako, M. Freyberg, M. J. et al. 2001, A\&̊A, 365, L174

Sakano, M., Warwick, R. S., Decourchelle, A. \& Predehl, P. 2003, Mon. Not. Roy. Astron. Soc., 340,747

Sakano, M., Warwick, R. S., Decourchelle, A. \& Predehl, P. 2004, Mon. Not. Roy. Astron. Soc., 350,129

Schoedel, R., Ott, T., Genzel, R., Hofmann, R. et al. 2002, Nature, 419, 694

Schoedel, R., Eckart, A., Iserlohe, C., Genzel, R. \& Ott, T. 2005, ApJ (Letter), 625, L111

Schulz, N. S., Canizares, C., Huenemoerder, D., Kastner, J. H., Taylor, S. C. \& Bergtrom, E. J. 2001, ApJ, 549, 441

Senda, A. 2004, PhD thesis, Kyoto University

Takagi, S., Murakami. H. \& Koyama, K. 2002, ApJ, 573, 275

Takahashi, H., Okada, Y., Kokubun, M., \& Makishima, K. 2004, ApJ, 615, 242

Tsujimoto, M., Koyama, K., Kobayashi, N., Saito, M., Tsuboi, Y. \& Chandler, C. J. 2004, Publ. Astron. Soc. Japan, 56, 341

Wang, Q. D., Gotthelf, E. V. \& Lang, C. C. 2002a, Nature, 415, 148

Wang, Q. D., Lu, F. \& Lang, C. C. 2002b, ApJ, 581, 1148

Weaver, K. A., Heckman, T. M., Strickland, D. K. \& Dahlem, M. 2002, ApJ (Letter) , 576, L19

Yamauchi, S., Koyama, K., Sakano, M. \& Okada, K. 1996, Publ. Astron. Soc. Japan, 48, 719

\section{Discussion}

PANNUTI: Have the three SNRs described in the talk been detected in the radio as well as the X-ray.

Koyama: G359 has been detected in the radio: The radio counterpart is shell-like. Another SNR, Sgr A East also has a well-known radio counterpart. The third SNR, G0.3, has not been detected in the radio. 


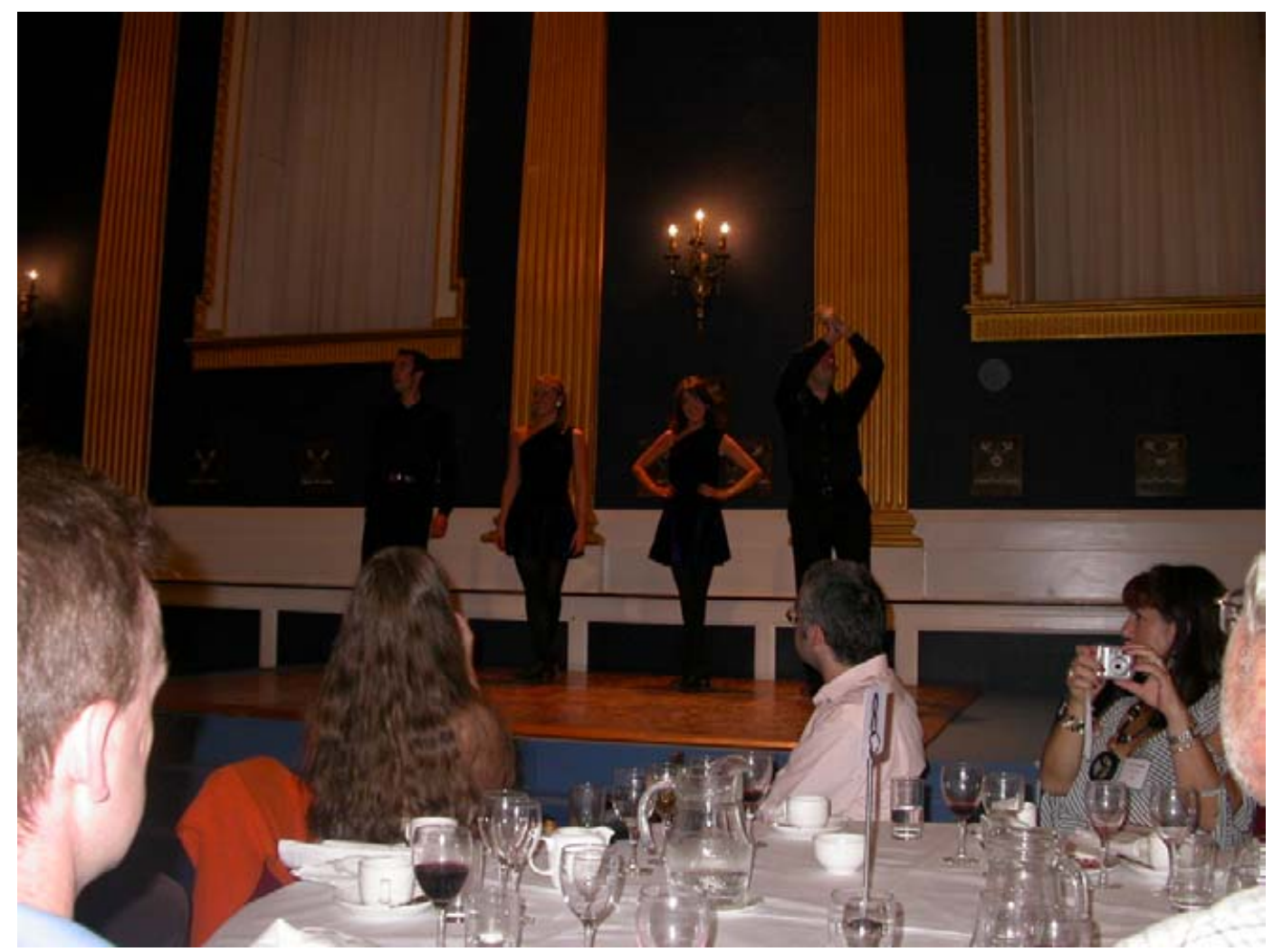

A demonstration of modern Irish dancing at the end of the Dinner. 